\title{
OSCILLATION OF SOLUTIONS OF IMPULSIVE NEUTRAL DIFFERENCE EQUATIONS WITH CONTINUOUS VARIABLE
}

\author{
GENGPING WEI AND JIANHUA SHEN
}

Received 21 June 2005; Revised 1 April 2006; Accepted 25 April 2006

We obtain sufficient conditions for oscillation of all solutions of the neutral impulsive difference equation with continuous variable $\Delta_{\tau}(y(t)+P(t) y(t-m \tau))+Q(t) y(t-l \tau)=0$, $t \geq t_{0}-\tau, t \neq t_{k}, y\left(t_{k}+\tau\right)-y\left(t_{k}\right)=b_{k} y\left(t_{k}\right), k \in \mathbb{N}(1)$, where $\Delta_{\tau}$ denotes the forward difference operator, that is, $\Delta_{\tau} z(t)=z(t+\tau)-z(t), P(t) \in C\left(\left[t_{0}-\tau, \infty\right), \mathbb{R}\right), Q(t) \in C\left(\left[t_{0}-\right.\right.$ $\tau, \infty),(0, \infty)), m, l$ are positive integers, $\tau>0$ and $b_{k}$ are constants, $0 \leq t_{0}<t_{1}<t_{2}<\cdots<$ $t_{k}<\cdots$ with $\lim _{k \rightarrow \infty} t_{k}=\infty$.

Copyright (c) 2006 Hindawi Publishing Corporation. All rights reserved.

\section{Introduction}

Let $\mathbb{R}$ denote the set of all real numbers. For any $a \in \mathbb{R}$, define $\mathbb{N}(a)=\{a, a+1, a+2, \ldots\}$. For any $t, \tau \in \mathbb{R}, r \in \mathbb{N}(1)$, define $\mathbb{N}(t-r \tau, t-\tau)=\{t-r \tau, t-(r-1) \tau, \ldots, t-\tau\}$.

Consider the neutral impulsive difference equation with continuous variable

$$
\begin{gathered}
\Delta_{\tau}(y(t)+P(t) y(t-m \tau))+Q(t) y(t-l \tau)=0, \quad t \geq t_{0}-\tau, t \neq t_{k}, \\
y\left(t_{k}+\tau\right)-y\left(t_{k}\right)=b_{k} y\left(t_{k}\right), \quad k \in \mathbb{N}(1),
\end{gathered}
$$

where $\Delta_{\tau}$ denotes the foreward difference operator, that is, $\Delta_{\tau} z(t)=z(t+\tau)-z(t), P(t) \in$ $C\left(\left[t_{0}-\tau, \infty\right), \mathbb{R}\right), Q(t) \in C\left(\left[t_{0}-\tau, \infty\right),(0, \infty)\right), m, l$ are positive integers, $\tau>0$ and $b_{k}$ are constants, $0 \leq t_{0}<t_{1}<t_{2}<\cdots<t_{k}<\cdots$ with $\lim _{k \rightarrow \infty} t_{k}=\infty$. Set $l_{0}=\max \{m, l\}$. For any $t_{0} \geq 0$, let $\phi_{t_{0}}=\left\{\varphi:\left[t_{0}-\left(l_{0}+1\right) \tau, t_{0}-\tau\right] \rightarrow \mathbb{R} \mid \varphi(t)\right.$ is piecewise continuous on $\left[t_{0}-\right.$ $\left.\left(l_{0}+1\right) \tau, t_{0}-\tau\right], \varphi(t)$ is finite for every $t \in\left[t_{0}-\left(l_{0}+1\right) \tau, t_{0}-\tau\right]$, the right and left limits $\varphi\left(t^{+}\right)$and $\varphi\left(t^{-}\right)$of $\varphi(t)$ exist for every $t \in\left(t_{0}-\left(l_{0}+1\right) \tau, t_{0}-\tau\right)$, and $\varphi\left(\left(t_{0}-\left(l_{0}+1\right) \tau\right)^{+}\right)$ and $\varphi\left(\left(t_{0}-\tau\right)^{-}\right)$exist $\}$.

Definition 1.1. For given $t_{0} \geq 0$ and $\varphi \in \phi_{t_{0}}$, a real-valued function $x(t)$ is said to be a solution of (1.1) satisfying the initial value condition

$$
x(t)=\varphi(t), \quad t \in\left[t_{0}-\left(l_{0}+1\right) \tau, t_{0}-\tau\right],
$$

if $x(t)$ is defined on $\left[t_{0}-\left(l_{0}+1\right) \tau, \infty\right)$ and satisfies (1.1) and (1.2).

Hindawi Publishing Corporation

International Journal of Mathematics and Mathematical Sciences

Volume 2006, Article ID 34232, Pages 1-7

DOI 10.1155/IJMMS/2006/34232 
2 Oscillation of impulsive difference equations

For given $t_{0} \geq 0$ and $\varphi \in \phi_{t_{0}}$, by means of the method of steps, the solution of (1.1) exists and is unique.

Definition 1.2. A solution of (1.1) is said to be oscillatory if it is neither eventually positive nor eventually negative. Otherwise, it is called nonoscillatory.

When $\left\{t_{k}\right\}=\phi$, that is, $\left\{t_{k}\right\}$ is an empty set, (1.1) reduces to the neutral difference equation

$$
\Delta_{\tau}(y(t)+P(t) y(t-m \tau))+Q(t) y(t-l \tau)=0, \quad t \geq t_{0}-\tau .
$$

The oscillatory behavior of difference equations with continuous variable without impulses has been investigated by some authors, see, for example, $[1-3,5,6]$. However, to the present time, there exists no literature on impulsive delay difference equations with continuous variable. The purpose of this note is to study the oscillatory behavior of solutions of (1.1). If there is a sequence $\left\{m_{k}\right\}$ of positive integers such that $m_{k} \rightarrow \infty$ as $k \rightarrow \infty$ and $b_{m_{k}} \leq-1$, then it is easily seen that every solution of (1.1) is oscillatory. Therefore, we always assume that $b_{k}>-1$ for all $k \in \mathbb{N}(1)$. Throughout this note, we will use the convention

$$
\prod_{\left\{t_{k}\right\} \cap \mathbb{N}(t-r \tau, t-\tau)=\phi}\left(1+b_{k}\right)^{-1} \equiv 1, \quad \prod_{\left\{t_{k}\right\} \cap \mathbb{N}(t-r \tau, t-\tau)=\phi}\left(1+b_{k}\right) \equiv 1,
$$

where $\phi$ is an empty set and $r \in \mathbb{N}(1)$.

\section{Main results}

We first introduce two lemmas due to [4]. We give the following hypothesis:

(H) $r$ is an integer, $p(n) \geq 0, n=0,1,2, \ldots, b_{k}>-1, k=1,2,3, \ldots,\left\{n_{k}\right\}$ is an infinite subset of $\mathbb{N}(1)$ satisfying $n_{1}<n_{2}<\cdots<n_{k}<\cdots$ with $\lim _{k \rightarrow \infty} n_{k}=\infty$.

Lemma 2.1. Let $(H)$ hold. Assume that

(i)

$$
\limsup _{n \rightarrow \infty} \prod_{n-r \leq n_{k} \leq n-1}\left(1+b_{k}\right)^{-1}<\infty
$$

(ii)

$$
\liminf _{n \rightarrow \infty} \sum_{\substack{i=n-r, i \notin\left\{n_{k}\right\}}}^{n-1} p(i) \prod_{n-r \leq n_{k} \leq n-1}\left(1+b_{k}\right)^{-1}>\left(\frac{r}{r+1}\right)^{r+1} .
$$

Then the discrete impulsive difference inequality

$$
\begin{gathered}
y(n+1)-y(n)+p(n) y(n-r) \leq 0, \quad n \in \mathbb{N}(0), n \neq n_{k}, \\
y\left(n_{k}+1\right)-y\left(n_{k}\right) \leq b_{k} y\left(n_{k}\right), \quad k \in \mathbb{N}(1),
\end{gathered}
$$

has no eventually positive solution. 
Lemma 2.2. Let $(H)$ hold and $r \geq 2$. Assume that

(i)

$$
\liminf _{n \rightarrow \infty} \prod_{n+1 \leq n_{k} \leq n+r-1}\left(1+b_{k}\right)>0
$$

(ii)

$$
\liminf _{n \rightarrow \infty} \prod_{n+1 \leq n_{k} \leq n+r-1}\left(1+b_{k}\right)<\infty
$$

(iii)

$$
\liminf _{n \rightarrow \infty} \sum_{\substack{i=n+1 \\ i \notin\left\{n_{k}\right\}}}^{n+r-1} p(i) \prod_{n+1 \leq n_{k} \leq n+r-1}\left(1+b_{k}\right)>\left(\frac{r-1}{r}\right)^{r} .
$$

Then the discrete impulsive difference inequality

$$
\begin{gathered}
y(n+1)-y(n)-p(n) y(n+r) \leq 0, \quad n \in \mathbb{N}(0), n \neq n_{k}, \\
y\left(n_{k}+1\right)-y\left(n_{k}\right) \leq b_{k} y\left(n_{k}\right), \quad k \in \mathbb{N}(1),
\end{gathered}
$$

has no eventually negative solution.

Theorem 2.3. Let $t_{k+1}-t_{k}=m \tau, b_{k}>0$, and $\left(1+b_{k}\right) P\left(t_{k}\right)=\left(1+b_{k-1}\right) P\left(t_{k}+\tau\right)$ for $k=$ $1,2,3, \ldots,-1<P(t)<0$ with $\inf _{t \in\left[t_{0}-\tau, \infty\right)} P(t)>-1, Q(t) \in C\left(\left[t_{0}-\tau, \infty\right),(0, \infty)\right)$. If

$$
\liminf _{t \rightarrow \infty} \sum_{\substack{i \in \mathbb{N}(t-l \tau, t-\tau) \\ i \notin\left\{t_{k}\right\}}} Q(i) \prod_{t_{k} \in \mathbb{N}(t-l \tau, t-\tau)}\left(1+b_{k}\right)^{-1}>\left(\frac{l}{l+1}\right)^{l+1},
$$

then every solution of (1.1) oscillates.

Proof. Suppose, on the contrary, there is a solution $y(t)$ of (1.1) which is eventually nonoscillatory. If $y(t)$ is a solution of (1.1), then $-y(t)$ is a solution of (1.1). Without loss of generality, we assume that $y(t)>0$ for $t \geq t_{N}-\left(l_{0}+1\right) \tau \geq t_{0}-\tau$, where $N$ is some positive integer. Let

$$
z(t)=y(t)+P(t) y(t-m \tau), \quad t \geq t_{N}-\tau
$$

For any $t \geq t_{N}-\tau$, by $(1.1)$ and $\left(1+b_{k}\right) P\left(t_{k}\right)=\left(1+b_{k-1}\right) P\left(t_{k}+\tau\right)$ for $k=1,2,3, \ldots$, we have

$$
\begin{gathered}
\Delta_{\tau} z(t)=-Q(t) y(t-l \tau)<0 \quad \text { for } t \notin\left\{t_{k}\right\} \\
z\left(t_{k}+\tau\right)-z\left(t_{k}\right)=b_{k} z\left(t_{k}\right) \quad \text { for } k=N, N+1, N+2, \ldots
\end{gathered}
$$

From (2.10), it follows that $z(t)$ strictly decreases on $\left\{t_{k}+\tau, t_{k}+2 \tau, \ldots, t_{k}+(m-1) \tau, t_{k+1}\right\}$ $(k=N, N+1, N+2, \ldots)$, and noting $(2.11)$, the seqence $\left\{z\left(t_{N}+n \tau\right)\right\}_{n=1}^{\infty}$ has only two 
cases: eventually positive or eventually negative. If $\left\{z\left(t_{N}+n \tau\right)\right\}_{n=1}^{\infty}$ is eventually negative, noticing

$$
z\left(t_{N}+n \tau\right)=y\left(t_{N}+n \tau\right)+P\left(t_{N}+n \tau\right) y\left(t_{N}+(n-m) \tau\right), \quad n=1,2,3, \ldots,
$$

then $y\left(t_{N}+n \tau\right)<-P\left(t_{N}+n \tau\right) y\left(t_{N}+(n-m) \tau\right)$ eventually holds for $n$. It follows that $0<$ $y\left(t_{N}+(n+j m) \tau\right)<-P\left(t_{N}+(n+j m) \tau\right) y\left(t_{N}+(n+(j-1) m) \tau\right)<P\left(t_{N}+(n+j m) \tau\right) P\left(t_{N}+\right.$ $(n+(j-1) m) \tau) y\left(t_{N}+(n+(j-2) m) \tau\right)<\cdots<(-1)^{j} \prod_{i=1}^{j} P\left(t_{N}+(n+i) \tau\right) y\left(t_{N}+n \tau\right)$. By condition $-1<P(t)<0$ with $\inf _{t \in\left[t_{0}-\tau, \infty\right)} P(t)>-1$, we have $y\left(t_{N}+(n+j m) \tau\right) \rightarrow$ $0(j \rightarrow \infty)$, that is, $\lim _{j \rightarrow \infty} y\left(t_{N}+j m \tau\right)=0$. Noticing $(2.12)$, we get $\lim _{j \rightarrow \infty} z\left(t_{N}+j m \tau\right)=$ 0 . This is a contradiction with the condition that $\left\{z\left(t_{N}+n \tau\right\}_{n=1}^{\infty}\right.$ is eventually negative and strictly decreases. If $\left\{z\left(t_{N}+n \tau\right)\right\}_{n=1}^{\infty}$ is eventually positive, then

$$
y\left(t_{N}+n \tau\right)>z\left(t_{N}+n \tau\right), \quad z\left(t_{N}+n \tau\right)>0 \text { for large } n .
$$

Let $t_{N+j}=t_{N}+n_{j} \tau, j=1,2,3, \ldots$ By (1.1), (2.9), and (2.11)-(2.13), we conclude that $\left\{z\left(t_{N}+n \tau\right\}_{n=1}^{\infty}\right.$ is an eventually positive solution of the following impulsive difference inequality:

$$
\begin{gathered}
\Delta z\left(t_{N}+n \tau\right)+Q\left(t_{N}+n \tau\right) z\left(t_{N}+(n-l) \tau\right)<0, \quad n \geq 1, n \neq n_{j}, \\
z\left(t_{N}+\left(n_{j}+1\right) \tau\right)-z\left(t_{N}+n_{j} \tau\right)=b_{N+j} z\left(t_{N}+n_{j} \tau\right), \quad j=1,2,3, \ldots,
\end{gathered}
$$

where $\Delta$ is the forward difference operator with respect to $n$. On the other hand, by condition (2.8), we have

$$
\liminf _{n \rightarrow \infty} \sum_{\substack{i=n-l \\ i \notin\left\{n_{j}\right\}}}^{n-1} Q\left(t_{N}+i \tau\right) \prod_{n-l \leq n_{j} \leq n-1}\left(1+b_{j}\right)^{-1}>\left(\frac{l}{l+1}\right)^{l+1} .
$$

Employing Lemma 2.1, we conclude that (2.14) has no eventually positive solution. This is a contradiction. Thus, the proof is complete.

Theorem 2.4. Let $t_{k+1}-t_{k} \equiv m \tau, b_{k}>0,\left(1+b_{k}\right) P\left(t_{k}\right)=\left(1+b_{k-1}\right) P\left(t_{k}+\tau\right)$ for $k=1,2$, $3, \ldots, m>l+1, P(t) \leq-1, Q(t) \in C\left(\left[t_{0}-\tau, \infty\right),(0, \infty)\right)$. If

$$
\begin{array}{r}
\liminf _{t \rightarrow \infty} \sum_{\substack{i \in \mathbb{N}(t-l \tau, t-\tau) \\
i \notin\left\{t_{k}\right\}}} Q(i) \prod_{t_{k} \in \mathbb{N}(t-l \tau, t-\tau)}\left(1+b_{k}\right)^{-1}>\left(\frac{l}{l+1}\right)^{l+1}, \\
\liminf _{t \rightarrow \infty} \sum_{\substack{i \in \mathbb{N}(t+\tau, t+(m-l-1) \tau) \\
i \notin\left\{t_{k}\right\}}} \frac{Q(i)}{-P(i+(m-l) \tau)} \prod_{t_{k} \in \mathbb{N}(t+\tau, t+(m-l-1) \tau)}\left(1+b_{k}\right)>\left(\frac{m-l-1}{m-l}\right)^{m-l},
\end{array}
$$

then every solution of (1.1) oscillates.

Proof. Suppose, on the contrary, there is a solution $y(t)$ of (1.1) which is eventually nonoscillatory. If $y(t)$ is a solution of (1.1), then $-y(t)$ is a solution of (1.1). Without 
loss of generality, we assume that $y(t)>0$ for $t \geq t_{N}-\left(l_{0}+1\right) \tau \geq t_{0}-\tau$, where $N$ is some positive integer. Set

$$
z(t)=y(t)+P(t) y(t-m \tau), \quad t \geq t_{N}-\tau
$$

For any $t \geq t_{N}-\tau$, by (1.1), we get

$$
\begin{gathered}
\Delta_{\tau} z(t)=-Q(t) y(t-l \tau)<0 \quad \text { for } t \notin\left\{t_{k}\right\}, \\
z\left(t_{k}+\tau\right)-z\left(t_{k}\right)=b_{k} z\left(t_{k}\right), \quad k=N, N+1, \ldots
\end{gathered}
$$

From (2.19), it follows that $z(t)$ strictly decreases on $\left\{t_{k}+\tau, t_{k}+2 \tau, \ldots, t_{k}+(m-1) \tau, t_{k+1}\right\}$ $(k=N, N+1, N+2, \ldots)$, and noting (2.20), the sequence $\left\{z\left(t_{N}+n \tau\right)\right\}_{n=1}^{\infty}$ has only two cases: eventually positive or eventually negative. If $\left\{z\left(t_{N}+n \tau\right)\right\}_{n=1}^{\infty}$ is eventually positive, noticing

$$
z\left(t_{N}+n \tau\right)=y\left(t_{N}+n \tau\right)+P(t) y\left(t_{N}+(n-m) \tau\right), \quad n=1,2,3, \ldots,
$$

then

$$
y\left(t_{N}+n \tau\right)>z\left(t_{N}+n \tau\right), \quad z\left(t_{N}+n \tau\right)>0 \text { for large } n
$$

Let $t_{N+j}=t_{N}+n_{j} \tau, j=1,2,3, \ldots$ By (1.1), (2.18), and (2.20)-(2.22), we conclude that $\left\{z\left(t_{N}+n \tau\right)\right\}_{n=1}^{\infty}$ is an eventually positive solution of the following impulsive difference inequality:

$$
\begin{gathered}
\Delta z\left(t_{N}+n \tau\right)+Q\left(t_{N}+n \tau\right) z\left(t_{N}+(n-l) \tau\right)<0, \quad n \geq 1, n \neq n_{j}, \\
z\left(t_{N}+\left(n_{j}+1\right) \tau\right)-z\left(t_{N}+n_{j} \tau\right)=b_{N+j} z\left(t_{N}+n_{j} \tau\right), \quad j=1,2,3, \ldots,
\end{gathered}
$$

where $\Delta$ is the forward difference operator with respect to $n$. On the other hand, by condition (2.16), we have

$$
\liminf _{n \rightarrow \infty} \sum_{\substack{i=n-l \\ i \notin\left\{n_{j}\right\}}}^{n-1} Q\left(t_{N}+i \tau\right) \prod_{n-l \leq n_{j} \leq n-1}\left(1+b_{j}\right)^{-1}>\left(\frac{l}{l+1}\right)^{l+1} .
$$

Employing Lemma 2.1, we conclude that (2.23) has no eventually positive solution. This is a contradiction. If $\left\{z\left(t_{N}+n \tau\right)\right\}_{n=1}^{\infty}$ is eventually negative, by simple calculation, we find that $z(t)$ satisfies

$$
\begin{gathered}
\Delta_{\tau} z(t)+P(t-l \tau) \frac{Q(t)}{Q(t-m \tau)} \Delta_{\tau} z(t-m \tau)+Q(t) z(t-l \tau)=0, \quad t \geq m \tau, t \neq t_{k}, \\
z\left(t_{k}+\tau\right)-z\left(t_{k}\right)=b_{N+j} z\left(t_{k}\right), \quad k=2,3, \ldots
\end{gathered}
$$


Let $t_{N+j}=t_{N}+n_{j} \tau$ for $j=1,2,3, \ldots$, then $\left\{z\left(t_{N}+n \tau\right)\right\}_{n=1}^{\infty}$ satisfies

$$
\begin{gathered}
\Delta z\left(t_{N}+n \tau\right)+P\left(t_{N}+n \tau-l \tau\right) \frac{Q\left(t_{N}+n \tau\right)}{Q\left(t_{N}+(n-m) \tau\right)} \Delta z\left(t_{N}+(n-m) \tau\right) \\
\quad+Q\left(t_{N}+n \tau\right) z\left(t_{N}+(n-l) \tau\right)=0, \quad n \geq m, n \neq n_{j}, \\
z\left(t_{N}+\left(n_{j}+1\right) \tau\right)-z\left(t_{N}+n_{j} \tau\right)=b_{N+j} z\left(t_{N}+n_{j} \tau\right), \quad j=2,3, \ldots,
\end{gathered}
$$

where $\Delta$ is the forward difference operator with respect to $n$. Noticing $\Delta z\left(t_{N}+n \tau\right)<0$ for $n \neq n_{j},\left\{z\left(t_{N}+n \tau\right)\right\}_{n=1}^{\infty}$ eventually satisfies the inequality

$$
\begin{gathered}
P\left(t_{N}+(n-l) \tau\right) \frac{Q\left(t_{N}+n \tau\right)}{Q\left(t_{N}+(n-m) \tau\right)} \Delta z\left(t_{N}+(n-m) \tau\right) \\
+Q\left(t_{N}+n \tau\right) z\left(t_{N}+(n-l) \tau\right)>0, \quad n \geq m, n \neq n_{j}, \\
z\left(t_{N}+\left(n_{j}+1\right) \tau\right)-z\left(t_{N}+n_{j} \tau\right)=b_{N+j} z\left(t_{N}+n_{j} \tau\right), \quad j=2,3, \ldots .
\end{gathered}
$$

And (2.27) is equivalent to the inequality

$$
\begin{gathered}
\Delta z\left(t_{N}+n \tau\right)+\frac{Q\left(t_{N}+n \tau\right)}{P\left(t_{N}+(n+m-l) \tau\right)} z\left(t_{N}+(n+m-l) \tau\right)<0, \quad n \geq m, n \neq n_{j}, \\
z\left(t_{N}+\left(n_{j}+1\right) \tau\right)-z\left(t_{N}+n_{j} \tau\right)=b_{N+j} z\left(t_{N}+n_{j} \tau\right), \quad j=2,3, \ldots
\end{gathered}
$$

So $\left\{z\left(t_{N}+n \tau\right)\right\}_{n=1}^{\infty}$ is an eventually negative solution of (2.28). On the other hand, noticing the condition (2.17), we have

$$
\liminf _{t \rightarrow \infty} \sum_{\substack{i=n+1 \\ i \notin\left\{n_{j}\right\}}}^{n+m-l-1} \frac{Q\left(t_{N}+i \tau\right)}{-P\left(t_{N}+(i+m-l) \tau\right)} \prod_{n+1 \leq n_{j} \leq n+m-l-1}\left(1+b_{j}\right)>\left(\frac{m-l-1}{m-l}\right)^{m-l} .
$$

By Lemma 2.2, (2.28) has no eventually negative solution. This is a contradiction. Thus, the poof is complete.

\section{Acknowledgment}

This work is supported by the NNSF of China (Grant no. 10571050) and the Science Foundation of the Education Committee of Hunan Province (Grant no. 04C457).

\section{References}

[1] G. Ladas, L. Pakula, and Z. Wang, Necessary and sufficient conditions for the oscillation of difference equations, Panamerican Mathematical Journal 2 (1992), no. 1, 17-26.

[2] J. Shen, Comparison theorems for oscillations of difference equations with continuous variables, Chinese Science Bulletin 41 (1996), no. 18, 1506-1510 (Chinese).

[3] J. Shen and I. P. Stavroulakis, Sharp conditions for nonoscillation of functional equations, Indian Journal of Pure and Applied Mathematics 33 (2002), no. 4, 543-554. 
[4] G. Wei, Oscillation of solutions of impulsive neutral delay difference equations, Mathematical Theory and Applications 19 (1999), no. 2, 119-121 (Chinese).

[5] Y. Zhang and J. Yan, Oscillation criteria for difference equations with continuous arguments, Acta Mathematica Sinica 38 (1995), no. 3, 406-411 (Chinese).

[6] B. G. Zhang, J. Yan, and S. K. Choi, Oscillation for difference equations with continuous variable, Computers \& Mathematics with Applications 36 (1998), no. 9, 11-18.

Gengping Wei: Department of Mathematics, Huaihua College, Huaihua, Hunan 418008, China E-mail address: weigengping@yahoo.com.cn

Jianhua Shen: Department of Mathematics, Hunan Normal University, Changsha, Hunan 410081, China

E-mail address: jhshen2ca@yahoo.com 


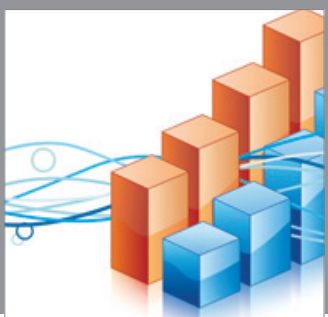

Advances in

Operations Research

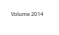

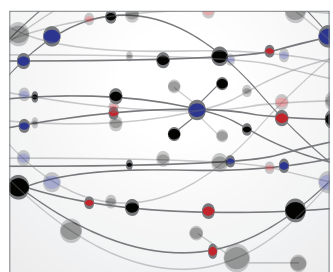

\section{The Scientific} World Journal
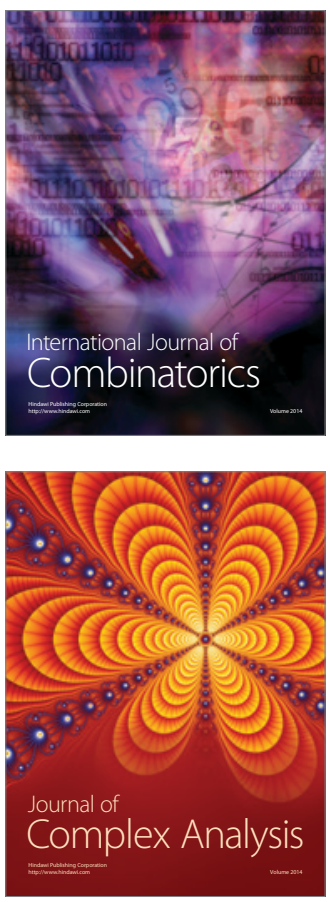

International Journal of

Mathematics and

Mathematical

Sciences
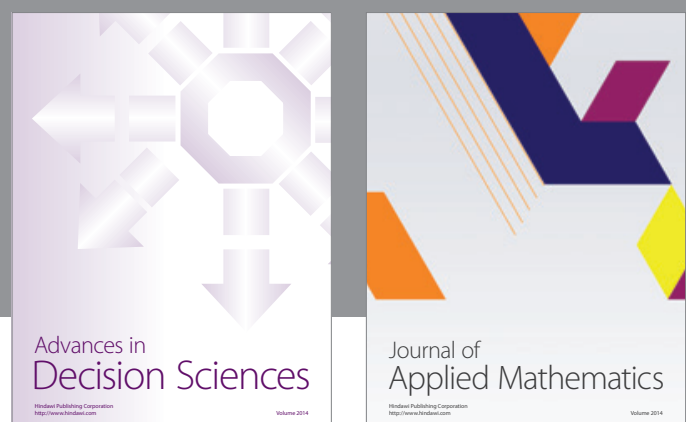

Journal of

Applied Mathematics
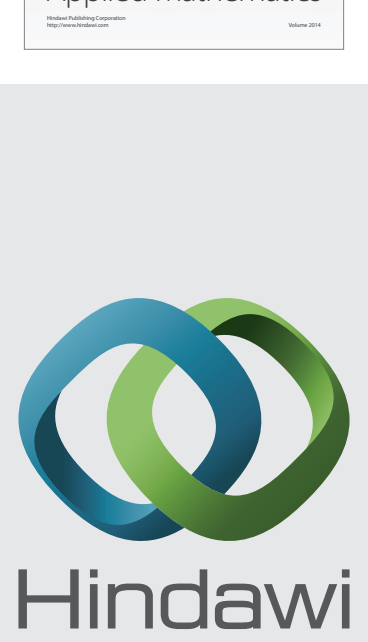

Submit your manuscripts at http://www.hindawi.com
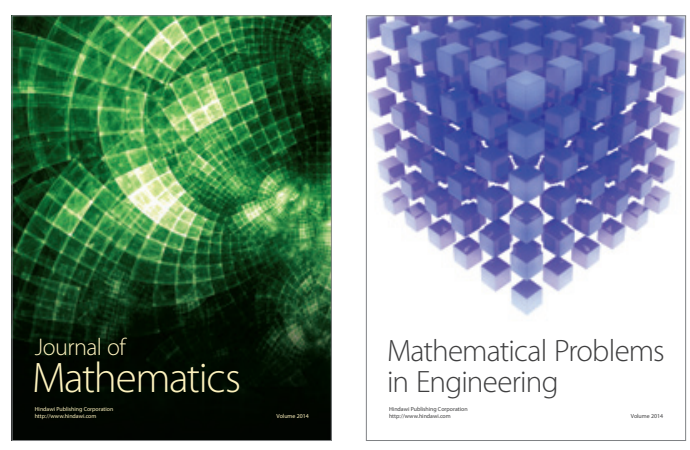

Mathematical Problems in Engineering
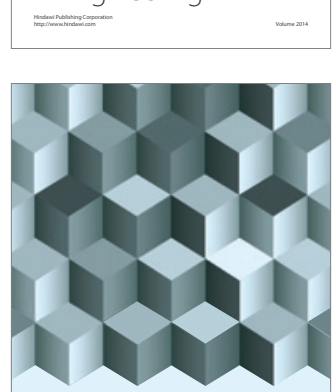

Journal of

Function Spaces
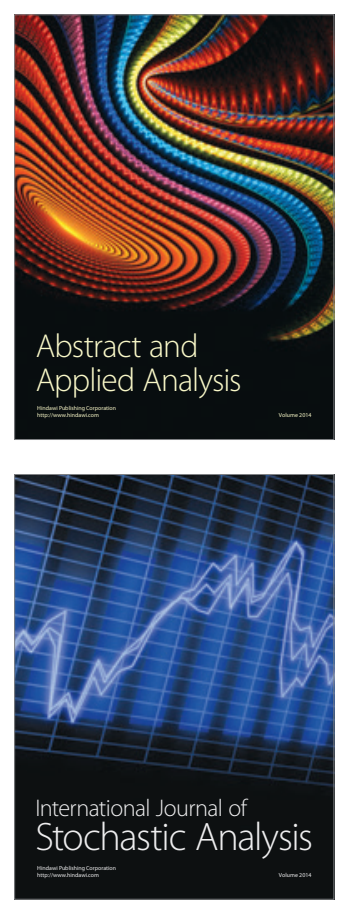

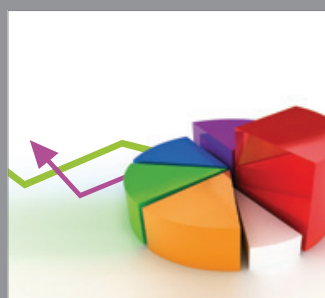

ournal of

Probability and Statistics

Promensencen
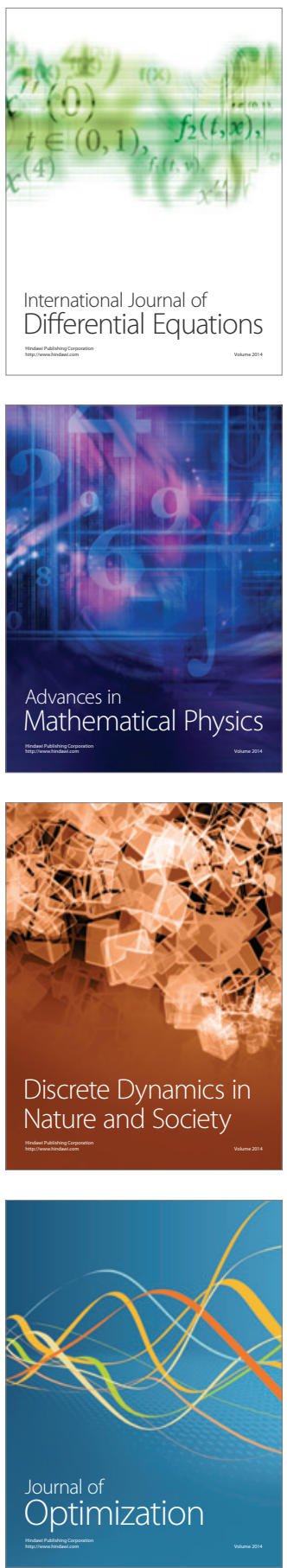